\title{
Maternal Linguistic Measures of Toddlers with Repaired Cleft Palate Following Early Intervention Program
}

\author{
M Pushpavathi*, Kavya V and Akshatha V \\ Department of Speech-Language Pathology, All India Institute of Speech and Hearing, India
}

Submission: July 17, 2017; Published: August 01, 2017

*Corresponding author: M Pushpavathi, Department of Speech-Language Pathology, All India Institute of Speech and Hearing (AIISH), Manasagangothri, Mysuru-570006, India, Tel: 0821-2502570; Email: shivanna.pushpa@gmail.com

\begin{abstract}
Maternal involvement plays an important role in early language intervention for children with repaired cleft lip and palate (RCLP) and aids in the development of speech and language. Research to document the usefulness of the same is limited. The present study attempts to provide an insight on the changes in maternal linguistic output by involving mothers in a parent-implemented early intervention program for toddlers with RCLP. The different maternal measures such as the total number of words, utterances, different words, mean length of utterance were investigated before and after 20 sessions of speech and language therapy. Six toddlers with RCLP and their mothers served as participants for the program. The baseline was established by measuring the parameters mentioned above. Speech and language therapy was given for 20 sessions with mothers as active participants. Mothers were oriented and counseled for speech stimulation. The post-therapy measurement was done after 20 sessions to analyze the linguistic output of the mothers. A nonparametric test was carried out due to the small sample size. Wilcoxon Signed Rank test was administered which emphasized that factors such as the total number of words, utterances, and different words showed a statistically significant difference $(\mathrm{p} \leq 0.05)$ between the pretest and posttest measures. The results revealed that the quantity of linguistic stimulation provided to toddlers by the mothers had vastly improved. The results highlight the importance of involving mothers as active participants in the early intervention program. It also illustrates the improvement in the implementation of language stimulation with intensive counseling and training.
\end{abstract}

Keywords: Cleft palate; Early Intervention Program; Parent-implemented; Maternal measures; Linguistic output

Abbreviations: RCLP: Repaired Cleft Lip and Palate; CLP: Cleft Lip and Palate; MLU: Mean Length of Utterance; FS: Focused Stimulation; EMT: Enhanced Milieu Teaching; TD: Typically Developing; ELIP: Early Language Intervention Program; AV: Audio-video; SALT: Systematic Analysis of Language Transcripts

\section{Introduction}

Cleft Lip and Palate (CLP) is a congenital abnormality of the face and oral cavity that occurs during the first trimester of pregnancy [1]. Management of CLP includes multiple intervention procedures to deal with issues such as facial abnormalities, difficulties in feeding, speech and language impairments, resonance and hearing difficulties [1-3]. International Peri-natal Database of Typical Oral Clefts revealed an overall prevalence of cleft lip with or without cleft palate was 9.92 per 10,000 [4]. The prevalence of cleft lip was 3.28 per 10,000 and that of cleft lip and palate was 6.64 per 10,000. In Indian sub-continent, out of the estimated 24.5 million births per year, the birth prevalence of clefts is somewhere between 27,000 and 33,000 clefts per year [5]. Another statistical survey by Times of India shows that the incidence of CLP has risen to 7 per 1,000 children. It was also reported that nearly 40,000 children are born in India every year with CLP [6].

Toddlers with repaired cleft lip and palate (RCLP) exhibit expressive language delay in addition to multiple associated problems which needs to be dealt by a team of experts [7]. During the developmental phase of speech and language toddlers with RCLP demonstrate features such as reduced mean length of utterance (MLU), reduced lexical diversity, smaller phonetic inventory and also a variety of deviant phonological processes [8]. Early language intervention is necessary for such children and several studies have clearly illustrated that early language intervention programs have been successful in facilitating both 


\section{Global Journal of Otolaryngology}

language and speech in children with CLP [9]. Various studies also emphasize constructive modifications in the speech and language skills of children when involved with parents/mothers in a structured parent-implemented program [10-12].

Research over last two decades have highlighted the associated problems seen in speech and language of children with CLP such as limited sound inventory, expressive language delay, limited vocabulary, reduced communication attempts, hyper nasality, nasal air emission, reduced speech intelligibility and compensatory articulation $[13,14]$. Such multiple associated problems need to be dealt by a team of experts from birth to 18 years of age. Several researchers have documented the need for early language intervention after the surgery for children with CLP and it has been proven that such intervention programs have been effective in facilitating both language and speech in such children [9]. The role of maternal speech stimulation in such early intervention programs has also been extensively studied $[14,15]$.

Early intervention for children with CLP focused mainly on aspects associated with speech production such as articulatory skills, resonance abilities and intelligibility. Recent studies and reviews have emphasized that children with CLP demonstrate poor expressive language abilities, when compared to their typically developing peers [14]. This highlights the fact that linguistic skills should also be focused in the early stages of intervention in children with CLP. However, there is no common agreement among SLPs and their techniques and programs that are being followed. Various hybrid models such as Enhanced Milieu Teaching (EMT), Focused Stimulation (FS) approach among others have been widely used over last two decades in children with CLP [9]. These models support promoting of language in natural conversation. The EMT approach applies behavioral principles to prompt functional language use in activities of daily living [16].

The Focused Stimulation approach is based on the naturalistic intervention model [17-19]. This approach emphasizes on techniques involving modeling and responsive interaction. The environmental arrangement and activities are organized in such a way that functional communication is taught to the child. This model assumes that language is learnt in a meaningful interactive setup with conversational partners who promote vocabulary building of the child [9]. From the early 1980's the spotlight of early intervention for children with communication disorders began to focus on the involvement of family members and its consequent effect on the child's speech and language output. One of the earlier studies examined the linguistic input of mothers interacting with toddlers with CLP [15]. Twenty six mother-child pairs involving 13 mother and cleft-child dyads were compared to their non-cleft counterparts. On examination of the data it was seen that mothers of children with CLP used more conversational devices such as modeling and expansion.
These mothers used lesser utterances than their counterparts and also had a smaller mean length of utterance (MLU) which indicates that they were inclined to simplify language and its structure to better suit their child's needs.

A previous study examined the effects of early intervention by training the parents/grandparents/nannies of children with various developmental disabilities using the Enhanced Milieu Teaching (EMT) approach [20]. The authors examined the changes in the communicative skills of the children as a result of a combined therapist and parent-implemented naturalistic approach. It was seen that most of the parents generalized the newly acquired skills to interactive sessions at home. Also more than $50 \%$ of the children showed significant improvements in measures of expressive language. The study has further highlighted the arrangements to be made for implementation of EMT and the skills required for teaching the parents or caregivers.

A similar and more recent study was conducted in order to determine whether parents could be trained to effectively deliver early intervention to children with CLP [21]. The study also aimed to examine whether intervention resulted in a reduction of articulatory errors and improvement in vocabulary and speech sound inventory. Ten mother-child dyads involving children with CLP were compared to mother-child pairs consisting of typically developing children. The results of the study revealed that postintervention there was a notable increase in the speech sound inventory, vocabulary and a reduction in the compensatory articulatory errors of children with CLP. Also with respect to the maternal measures it was seen that there was considerable improvement in the number of utterances, words and MLU. The MLU of mothers of children with CLP was found to be lesser than their counterparts, which suggests that these mothers were more in tune with their children's level of communication and used sentences with simple structures according to the child's level.

In the Indian scenario, limited studies have been carried out highlighting the relationship between early intervention and the speech and language output of children with CLP. Also there is a dearth of studies in India which investigate the effectiveness of a structured parent-implemented program for young children with CLP. Some authors investigated the nature of stimulation provided by mothers of typically developing (TD) children in the age range of 3-5 years [22]. Mohite's Home Environment Inventory was administered before and after the intervention sessions in order to judge the stimulation provided by mothers. The mothers in the experimental group were exposed to training for 16 weeks while the control group sample was not exposed to any kind of program. Post-intervention it was discovered that mothers in the experimental group were significantly better in providing stimulation to their children when compared to their counterparts. 
Therefore, it is apparent that maternal speech stimulation and parent-implemented program plays an extremely important role in development of speech and language. The efficacy of treatment in settings other than the clinical setup is critical for successful remediation and thus, educating the mother who is mostly the conversational partner with the child, is warranted. Although several approaches have been proposed in the western context to improve the language abilities of children with CLP, very few studies in the Indian context have focused on investigating the long term effects of language intervention strategies in this group. Hence, the present study attempts to provide an insight on the efficacy of speech and language therapy using focused stimulation approach by involving mothers and also aims to describe the speech and language characteristics of toddlers with CLP before and after speech-language intervention. The objectives of the present study are as follows:

a. To study the impact of a parent-implemented intervention program on maternal measures such as the total number of words, utterances and different words.

b. To investigate the changes if any, in the mean length of utterance (MLU) before and after the intervention program.

\section{Method}

\section{Participants}

Six toddlers diagnosed as Expressive Language Delay secondary to repaired cleft lip and palate, along with their mothers, served as participants for the study. The toddlers considered were in the age range of 2-3 years and did not have any associated conditions. They also had normal auditory and cognitive abilities. The age, educational qualifications and socio-economic status of the mothers were controlled as much as possible. The mothers were in the age range of 20-35 years and had at least passed the $12^{\text {th }}$ grade in terms of educational qualification. The families belonged to the middle to high socio-economic status. The native language of the parent-child dyads was Kannada. The speech and language evaluation of the toddlers was carried out by a qualified Speech Language Pathologist. An informed consent (approved by the Ethical Committee) was taken from the parents that outlined the details of the intervention procedure. Following this, the participants were registered for the Early Language Intervention Program (ELIP) at the Unit for Structural and Oro-facial Anomalies in our institute.

\section{Enrollment to the Early Intervention Program}

Subsequent to enrollment of the participants in the Early Language Intervention Program, a questionnaire was administered on the mothers that assessed the awareness of mothers on issues related to cleft lip and palate. The questionnaire which was developed in our institute assessed the levels of awareness present in mothers on various aspects related to CLP such as causes, associated problems, assessment, surgery, treatment and speech therapy [23]. This was followed by a detailed orientation provided to the mothers covering the various issues related to children with CLP.

\section{Data collection}

Prior to beginning the intervention program, a pretherapeutic assessment was carried out in order to ascertain the baseline speech and language skills present in the child. Audiovideo (AV) recording was carried out for each dyad during the initial interactions in order to establish the amount of linguistic stimulation provided by the mother. The maternal measures such as

\section{a. Number of total words used \\ b. Number of words and different words \\ c. Mean length of utterance (MLU) were also profiled.}

The AV recordings were carried out in a quiet room during unstructured and free play sessions between the mother and the child. The mother-child interaction was documented using a Handy cam recorder (Sony DCR-SR88). The recorder was placed on a tripod stand at a distance of approximately 8-10 feet from the child in such a way that the entire play area was captured and also making sure that the child was not distracted. The duration of each recording was approximately 45 minutes when the child was co-operative. In instances wherein the child was not co-operative various strategies such as a few trial recordings were carried out or environmental modifications were done. The mothers were oriented and counseled regarding the importance and ways of providing speech stimulation. Following this, a total number of 20 therapy sessions for each child were conducted by an SLP and the mother was also encouraged to be an active participant. Post-therapy the $20^{\text {th }}$ session was recorded wherein the mother was asked to provide linguistic stimulation to her child. Thus 2 recordings were done for each mother-child dyad and in total, 12 video recordings and analyses were carried out.

\section{Speech and Language Intervention}

The speech-language therapeutic sessions were carried out in a clinical setup by a qualified and experienced SLP and each session lasted about forty-five minutes. A master lesson plan was prepared for each child by taking up specific goals and activities, based on the baseline which was ascertained during the pre-therapeutic assessment. The needs of each child were determined through direct observation and interviewing the mother. Goals with respect to resonance such as increasing the oral airflow and improving the oro motor abilities, speechrelated goals such as increasing the consonant inventory and linguistic goals such as increasing the functional and lexical vocabulary were taken up. An interactive play way method was used to improve vocabulary, vowel and consonant inventory and to increase the frequency of meaningful utterances.

Focused stimulation approach was demonstrated to each 
mother during therapeutic sessions using low-cost materials and commonly available toys. A multisensory approach was used to increase the oromotor movements. Visual feedback using mirror work and tactile cues with interesting oromotor activities was provided appropriately wherever required. A corpus of vocabulary was prepared in the master lesson plan which contained the most commonly used functional words by toddlers. This was done with the aim of increasing the child's functional vocabulary. The positive behaviors and behaviors which were attempted correctly by the child were reinforced to increase the co-operation and confidence in doing the activity. The mother was given specific goals and told to carry out a similar intensive speech stimulation and facilitation at home by using modeling, imitation and demonstration techniques in the absence of direct supervision of the SLP.

\section{Data Analysis}

Each of the 45 minute session of mother-child interaction was transcribed using International Phonetic Alphabet (IPA) symbols in the Baraha 9.1 software and analyzed. A PretestPosttest design was used in order to monitor the changes in maternal measures between the baseline and the $20^{\text {th }}$ session. With respect to the maternal linguistic inventory, parameters such as total number of words, total number of utterances, total number of different words and mean length of utterance (MLU) were calculated in the pre- and post-therapy conditions. The transcribed maternal data was analyzed using the Systematic Analysis of Language Transcripts (SALT) software [24]. The software calculates the number of words by counting the total number of complete words. An utterance refers to a statement which may contain one or many words. The total number of different words is calculated by the number of different word roots. The MLU is calculated by computing the number of words in each utterance. The tabulated data was then entered in a Microsoft excel sheet and the difference in the frequency of child and mother's parameters were calculated and statistically analyzed. Non-parametric test (Wilcoxon Signed Rank test) was carried out due to the small sample size.

\section{Results}

The present study is one of the preliminary attempts to highlight the importance of actively involving mothers of toddlers with CLP, in a parent-implemented speech and language intervention program. The Pretest-Posttest measures with respect to maternal linguistic inventory were compared and the findings obtained have been highlighted below.

\section{Effect of Intervention on the Vocabulary of Mothers}

In order to scrutinize whether intensive speech and language therapy brings about any positive effects on the maternal lexical output, measures such as the total number of utterances, total number of words and the total number of different words were obtained. The values obtained for the pre- and post-test measures of the three parameters have been tabulated as shown.



Figure 1: Frequency of total utterances across participants in pre- and post-test conditions ( $\mathrm{BL}-$ Baseline; $\mathrm{S} 20-20^{\text {th }}$ session).

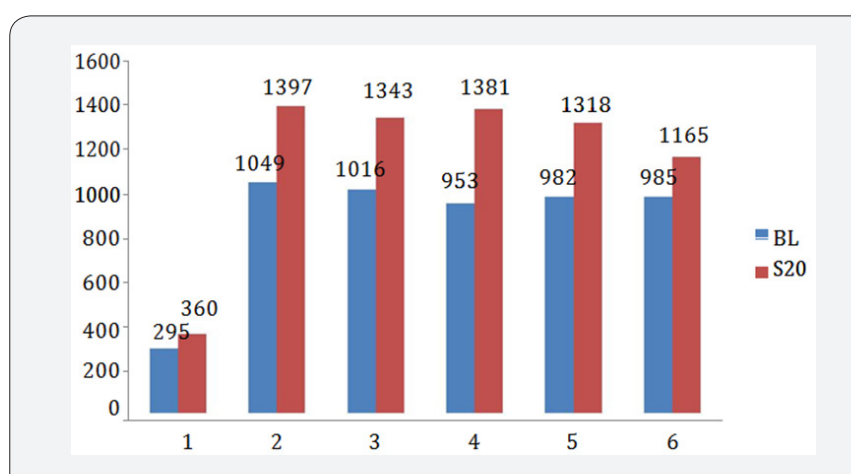

Figure 2: Frequency of total words across participants in preand post-test conditions ( $\mathrm{BL}-$ Baseline; $\mathrm{S} 20-20^{\text {th }}$ session).

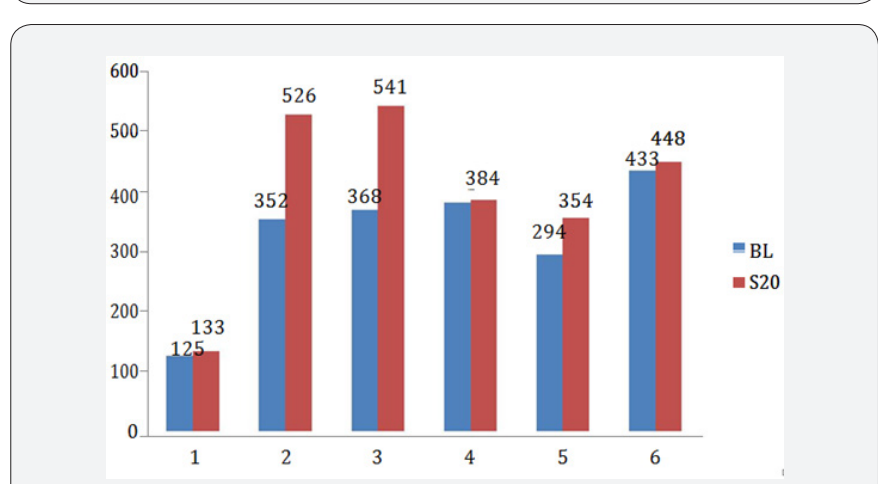

Figure 3: Frequency of total number of different words across participants in pre- and post-test conditions (BL - Baseline; S20 $-20^{\text {th }}$ session).

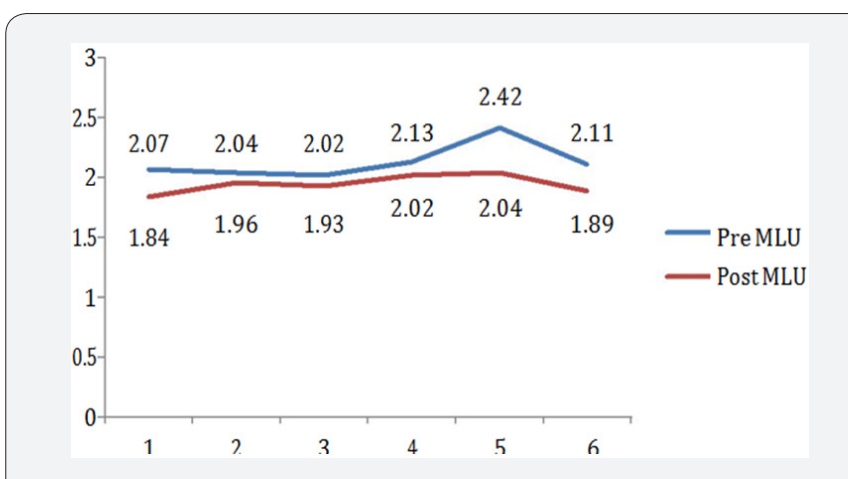

Figure 4: Trend of Mean Length Utterance of the participants in pre- and post-therapy conditions. 
The three bar graphs (Figures 1-3) given above portray the frequency of utterances, words and different words used by the mothers in pre-therapy and post-therapy conditions. The graphs clearly show that the frequency of utterances, words and different words showed a prominent increase between baseline and the $20^{\text {th }}$ session. In order to statistically analyze the data, Wilcoxon Signed Rank test was administered for the maternal measures which emphasized that all the three measures showed a statistically significant difference $\left({ }^{*} \mathrm{p} \leq 0.05\right)$ between the preand post-test measures which is shown in Table 1 below.

Table 1: Statistical analysis of maternal measures comparing pre- and post-test conditions $\left({ }^{*} p \leq 0.05\right)$.

\begin{tabular}{|c|c|c|c|c|c|}
\hline $\begin{array}{c}\text { Maternal } \\
\text { Measure }\end{array}$ & \multicolumn{2}{|c|}{ Mean } & \multicolumn{2}{c|}{ S.D. } & P value \\
\hline & Pre & Post & Pre & Post & \\
\hline Total utterances & 477.67 & 661.67 & 150.08 & 240.24 & $0.03^{*}$ \\
\hline Total words & 880.00 & 1160.67 & 288.46 & 400.87 & $0.03^{*}$ \\
\hline $\begin{array}{c}\text { Total different } \\
\text { words }\end{array}$ & 325.33 & 397.67 & 107.91 & 149.46 & $0.03^{*}$ \\
\hline
\end{tabular}

\section{Effect of intervention on the linguistic productivity of mothers}

The line graph given (Figure 4) shows the mean length of utterance (MLU) of the six mothers before therapy and in the $20^{\text {th }}$ session. The trend clearly shows that in the post-therapy, i.e. in the $20^{\text {th }}$ session, the MLU of all the six mothers showed a marginal reduction. As seen in Table 1 , in the baseline session the MLU of the mothers ranged from 2.02 to 2.42, whereas 20 sessions later the values showed a slight drop, ranging from 1.84 to 2.04. These changes noted in terms of the different maternal measures have been discussed in detail in the section below.

\section{Discussion}

The results of the present study clearly depict that there was a remarkable increase in the lexical output of the mothers with respect to the frequency of words and utterances from the baseline to post-therapy. The results indicated two interesting points with reference to maternal lexical measures. The first interesting point observed was reduced speech output among the mothers of children with RCLP prior to attending therapy. The mothers showed a tendency to use one-word utterances and non-verbal means such as meaningful gestures and actions while communicating with the child. This finding is in concordance with a previous study which has documented that mothers of children with CLP show a tendency to use fewer utterances and words in an attempt to simplify their linguistic output [15]. This may be due to various factors such as lack of awareness regarding the need for early linguistic stimulation, frequent hospitalizations due to recurring health issues and underestimating the potential of the child with RCLP in terms of their linguistic abilities. Especially in the Indian context some parents believe that oro facial malformations such as CLP are a major handicap and that the child may not be able to develop adequate speech and language skills. The mothers and family as a whole show a tendency to pamper the child as they do not understand the long term beneficial effects of linguistic stimulation at an early age. They may also feel that the children may be taxed as they are not competent enough to cope at such a young age.

The second observation made from the study was improved speech output of the mothers after attending early speech and language intervention program. It can be noted that the increase in frequency for utterances and words are slightly higher when compared to the number of different words. This highlights the fact that mothers showed a greater lexical diversity post-therapy. These results support the findings of an earlier study wherein it was seen that after receiving training, mothers of children with CLP showed an increase in the total number of words, different words and utterances when interacting with their children [21]. Therefore, providing training to mothers using previously used approaches such as the Focused Stimulation Approach may change the way in which they interact with their children by varying the number and variety of different words and utterances used.

With respect to the MLU, the post-therapy analyses revealed that there was a decline when compared to the baseline session. These findings support similar studies which reported that mothers of children with clefts tended to use smaller utterances and had a smaller MLU when compared to the mothers of typically developing children [15,21]. The authors also reported that mothers of typically developing children used more complex language than mothers of children with CLP. These mothers showed a tendency to use simpler language during interactions with children [21]. They also noted that the mothers used more responsive labels and expansion strategies to communicate with their children.

Especially in the Indian context, there may be a tendency for the mothers to simplify the content and structure of language due to lack of awareness about the necessity of intensive speech and language training for children with CLP in the formative years as well as due to associated superstitious beliefs such as the child may be too young to begin speaking or that too much training may be taxing for the child. Higher MLU has been conventionally linked to greater linguistic efficiency in case of children. This would lead us to believe that the same applies to mothers as well. It is interesting to note that in the present study, mothers of toddlers with CLP tended to use a lesser MLU which indicates that probably there has been a tendency to use a simpler linguistic structure in order to elicit a better output from their children. They have also possibly tried the analogy of consciously getting down to the level of their children by reducing the complexity of the language used. This also suggests that the mothers are learning to develop better interaction patterns with the children post-intervention by observing and putting 


\section{Global Journal of Otolaryngology}

to use the strategies depicted by the SLP. These findings could also be correlated with previous findings which reported that mothers of children with CLP tended to use a different pattern of interaction wherein they used more of non-verbal means and actions to maintain the child's attention when communicating about objects and the surroundings [25].

In essence, the present study highlights the positive changes seen in mothers of children with RCLP, which were brought about by an intensive and regular parent-implemented early intervention program. With respect to maternal linguistic output, the results revealed that the quantity of speech stimulation provided to children with CLP by the mothers had vastly improved. There was a significant increase in the total number of words, different words and utterances of the mothers post-intervention. They were also seen to use simplification of language which was seen in the reduction in mean length of utterance. This clearly reveals the need for involving and training the mothers of children with RCLP by trained professionals in order to bring about positive changes in the speech and language of children with CLP.

\section{Conclusion}

Thus the results implicate the necessity to begin intervention at the earliest possible age in this population since adequate attention has not been paid for language acquisition in these children followed by rehabilitation. In this perspective the present study highlights the importance of regular home training and stimulation by parents in order to obtain a positive speech and language output. In India the importance and urgency of attending regular therapy and the consequences of stimulating the child during the critical years is yet to be understood by the general population. By conducting studies like the present one, it is essential to eliminate the social stigma attached to CLP and all the parameters associated with it. The findings of the present study will help parents realize the need for a parent-implemented interaction for the child during the formative period and hopefully bring about positive changes in the outlook of parents regarding speech and language training. It will also facilitate the process of continued early intervention for the toddlers and by taking up the goals of speech therapist the intervention program can be implemented by mothers in regions where there is a lack of such facilities.

\section{Acknowledgement}

This is a part of ongoing research on "Efficacy of Early Language Intervention Program for Children with Repaired Cleft lip and Palate", funded by the Department of Science and Technology (DST - No. SB/SO/HS/02/2014), Government of India. The authors would like to thank DST for funding the project. The authors would also like to thank Dr. S. R. Savithri, Director, All India Institute of Speech and Hearing, Mysore, for providing the infrastructure to carry out the study.

\section{References}

1. Kummer AW (2008) Cleft palate and craniofacial anomalies: Effects on speech and resonance. Delmar Cengage Learning, New York, USA pp. $1-772$.

2. Baker SR, Owens J, Stern M, Willmot D (2008) Coping strategies and social support in the family impact of cleft lip and palate and parents adjustment and psychological distress. Cleft Palate Craniofac J 46(3): 229-236.

3. Kapp-Simon KA, \& Krueckeburg S (2000) Mental developmental in infants with cleft lip and/or palate. Cleft Palate Craniofac J 37(1): 6570.

4. IPDTOC (2011) Prevalence of birth of cleft lip with or without cleft palate: Data from the international perinatal database of typical oral clefts. Cleft Palate Craniofac J 48 (1): 66-78.

5. Mossey P, Little J (2009) Addressing the challenges of cleft lip and palate research in India. Indian J Plast Surg 42(Suppl): S9-S18.

6. Shrivatsav S (2013) Parents still don't bring cleft lip, palate kids for treatment. Times of India. Nagpur, India.

7. Scherer NJ (1999) The speech and language status of toddlers with cleft lip and/ or palate following early vocabulary intervention. Am J Speech Lang Pathol 8: 81-93.

8. Morris H, Ozanne A (2003) Phonetic, phonological and language skills of children with a cleft palate. Cleft Palate Craniofac J 40(5): 460-470.

9. Scherer NJ, Kaiser A (2007) Early intervention in children with cleft palate. Infants Young Child 20: 355-366.

10. Goudy S, Ingraham C, Canady J (2011) The occurrence of velopharyngeal insufficiency in Pierre Robin Sequence patients. Int J Pediatr Otorhinolaryngol 75(10): 1252-1254.

11. Pamplona MC, Ysunza A, Espinosa J (1999) A comparative trial of two modalities of speech intervention for compensatory articulation in cleft palate children, phonologic approach versus articulatory approach. Int J Pediatr Otorhinolaryngol 49(1): 21-26.

12. Pamplona MC, Ysunza A, Ramirez P (2004) Naturalistic intervention in cleft palate children. Int J Pediatr Otorhinolaryngol, 68(1): 75-81.

13. Sunitha R, Jacob M, Jacob MS, Nagarajan R (2004) Providing intervention services for communication deficits associated with cleft lip and/or palate- A retrospective analysis. Asia Pacific Disability Rehabilitation Journal 15: 78-85.

14. DAntonio LL, Scherer NJ (2008) Communication Disorders Associated with Cleft Palate. In J Losee , R Kirschner (eds.), Comprehensive Cleft Care Mc Graw: Hill, New York, USA.

15. Chapman KL, Hardin MA (1991) Language input of mothers interacting with their young children with cleft lip and palate. Cleft Palate Craniofac J 28(1): 78-85.

16. Kaiser AP, Hancock TB, Hester PP (1998) Parents as co interventionists: Research on applications of naturalistic language teaching procedures. Infants Young Child 10(4): 46-55.

17. Girolametto LE (1988) Improving the social-conversational skills of developmentally delayed children. J Speech Hear Disord 53(2): 156167.

18. Girolametto LE, Pearce P, Weitzman E (1997) Effects of lexical intervention on the phonology of late talkers. J Speech Hear Res 40(2): 1274-1283.

19. Girolametto LE, Pearce P, Weitzman E (1996) Interactive focused stimulation for toddlers with expressive vocabulary delays. J Speech Hear Res 39(6): 1274-1283.

20. Kaiser AP, Hancock TB (2003) Teaching parents new skills to support 
their young children's development. Infants Young Child 16(1): 9-21.

21. Scherer NJ, DAntonio L, McGahey H (2008) Early intervention for speech impairments in children with cleft palate. Cleft Palate Craniofac J 45: 18-31.

22. Manocha A, Narang D, Balda S (2008) Role of intervention in improving maternal stimulation level. J Soc Sci 17(2): 181-184.

23. Thammaiah I, Jasmine L, Pushpavathi M (2012) Awareness of parents on the nature of cleft lip and palate: An exploratory study. JAIISH 30 : 15-22.

24. SALT (2010) Systematic analysis of language transcripts (V. Bilingual SE) [Computer software and manual]. Madison, WI: Language Analysis Laboratory, University of Wisconsin-Madison, USA.

25. Wasserman GA, Allen R, Linares LO (1988) Maternal interaction and language development in children with and without speech-related anomalies. J Commun Disord 21(4): 319-331. 